BOUNDARY VALUE PROBLEMS FOR THE

STEADY BOLTZMANN EQUATION

R. Illner and J. Struckmeier

DMS-708-IR

July 1995 


\title{
Boundary Value Problems for the Steady Boltzmann Equation
}

\author{
R. Illner and J. Struckmeier* \\ Department of Mathematics and Statistics \\ University of Victoria \\ P.O. Box 3045 \\ Victoria, B.C. \\ Canada V8W 3P4
}

\begin{abstract}
We discuss steady boundary value problems for the Boltzmann equation with inflow and diffusive boundary conditions in 1, 2 and 3 dimensions, with suitable truncations of the collision kernel. General existence and uniqueness results are obtained if the domain is sufficiently small. In one dimension, the existence of solutions on general intervals is obtained by abstract fixed point theory. A-priori estimates for solutions in more than one dimension and some numerical examples are also given.
\end{abstract}

\section{Introduction}

We consider the steady Boltzmann equation

$$
v \cdot \nabla_{x} f=Q(f), \quad x \in \Omega, v \in \mathbb{R}^{3}
$$

on the multidimensional domain $\Omega \subset \mathbb{R}^{n}$ with various boundary conditions on $\partial \Omega$ and $n=1,2$ or 3. Here $Q(f)$ denotes the collision operator written in the form

$$
Q(f)=Q_{+}(f)-f L(f) \text {. }
$$

where $Q_{+}(f)$ stands for the gain term, $f L(f)$ for the loss term due to binary collision of gas particles,

$$
\begin{aligned}
Q_{+}(f) & =\iint_{R^{3} S_{+}} B\left(\left|v-v_{*}\right|, n\right) f\left(v^{\prime}\right) f\left(v_{*}^{\prime}\right) \mathrm{d} n \mathrm{~d} v_{*} \\
L(f) & =\int_{R^{3}} \int_{S_{+}} B\left(\left|v-v_{*}\right|, n\right) f\left(v_{*}\right) \mathrm{d} n \mathrm{~d} v_{*}
\end{aligned}
$$

- Permanent address: Department of Mathematics, University of Kaiserslautern, P.O. Box 3049,67653 Kaiserslautern, Germany. 
and $S_{+}$is the hemisphere corresponding to $\left(v-v_{*}, n\right)>0$. The pair $\left(v^{\prime}, v_{*}^{\prime}\right)$ is given by the collision transformation

$$
J:\left(v, n, v_{*}\right) \rightarrow\left(v^{\prime},-n, v_{*}^{\prime}\right)
$$

by

$$
\begin{aligned}
v^{\prime} & =v-n\left(v-v_{*}, n\right), \\
v_{*}^{\prime} & =v_{*}+n\left(v-v_{*}, n\right) .
\end{aligned}
$$

Unless mentioned otherwise we assume the following kind of interaction law for binary collisions: For $v \in \mathbb{R}^{3}$ and $n \in S_{+}^{2}$, we assume that

$$
B\left(\left|v-v_{*}\right|, n\right)=\left|v-v_{*}\right|^{k} h(\theta),
$$

where $\theta$ is the polar angle of $n$ relative to a polar axis in direction $v-v_{*}$. The function $h$ is assumed to be integrable on $[0, \pi]$ with $\int_{S_{+}} h(\theta) \mathrm{d} n=1$ and the integer $k$ is chosen out of the set $\{-1,0,1\}$, which describes Maxwellian molecules $(k=0)$, hard-sphere gas $(k=1)$ and soft-sphere gas $(k=-1)$. Hence we cover three classical examples of molecular interaction laws.

In Section 2, we use the Kaniel-Shinbrot iteration scheme (see [1]) to prove a general existence and uniqueness result for a truncated problem; the collision kernel is modified such that collisions in which one of the (pre- or post-collisional) velocities has modulus less than $\varepsilon$ are disregarded, and the size of the domain must be bounded in terms of $\varepsilon$.

Subsection 2.2 deals with precribed influx boundary conditions, whereas partial results on diffusive boundary conditions are presented in Subsection 2.3. In this subsection, the Albedo operator is introduced and used to reduce the boundary value problem to a suitable fixed point operator.

In Section 3, we generalize the existing global existence results for one-dimensional slabs from Refs. [2] and [3] to purely diffusive boundary conditions.

Finally, in Section 4, we derive some a priori estimates for the full, 2- or 3-dimensional boundary value problem for the steady Boltzmann equation, in the hope that these estimates will eventually turn out to be useful for existence theorems. The last section is devoted to numerical examples.

\section{Local Existence Results}

\subsection{Truncation of the Collision Kernel}

Due to the singular limit $v=0$ of equation (1.1) we have to eliminate collisions between particles with small velocities. A formal solution of (1.1) can be derived by integrating along a characteristic line starting at some boundary point $x \in \partial \Omega$. The reason to eliminate small velocities from the collision process is to get an upper bound on the 'travelling time' of a particle through the domain $\Omega$.

We introduce the following truncation of the collision kernel $B$ : for $\varepsilon>0$ arbitrary but fixed let

$$
B_{\varepsilon}\left(v, v_{*}, n\right)= \begin{cases}c_{\varepsilon}\left|v-v_{*}\right|^{k} h(\theta) & \text { if } \min \left\{|v|,\left|v_{*}\right|,\left|v^{\prime}\right|,\left|v_{*}^{\prime}\right|\right\}>\varepsilon \\ 0 & \text { otherwise }\end{cases}
$$

with $c_{\varepsilon}>0$ such that $\int_{S_{+}} B_{\varepsilon}\left(v, v_{*}, n\right) \mathrm{d} n=\left|v-v_{*}\right|$ and $k \in\{-1,0,1\}$. Further, let $Q^{\varepsilon}$ be the collision operator with $B$ replaced by $B^{\varepsilon}$. 
The local proof is based on some a-priori estimates on $L(f)$ where $f$ is a Maxwellian distribution. For the collision kernels $B$ and $B^{\varepsilon}$ given above we find

Proposition 2.1 Assume that $f$ is a (normalized) Maxwellian

$$
f(v)=\left(\frac{\beta}{\pi}\right)^{3 / 2} \mathrm{e}^{-\beta v^{2}}, \quad v \in \mathbb{R}^{3}
$$

then

$$
L(f)(v)= \begin{cases}1 & \text { if } k=0 \\ \frac{\operatorname{erf}(\sqrt{\beta}|v|)}{|v|} & \text { if } k=-1 \\ \frac{1}{\sqrt{\beta \pi}} \mathrm{e}^{-\beta v^{2}}+\left(\frac{1}{2 \beta}+|v|^{2}\right) \frac{\operatorname{erf}(\sqrt{\beta}|v|)}{v \mid} & \text { if } k=1\end{cases}
$$

\section{Proof}

Because $f$ is a (normalized) Maxwellian, we obtain using spherical coordinates

$$
L(f)(v)=2 \pi\left(\frac{\beta}{\pi}\right)^{3 / 2} \int_{0}^{\infty} \int_{-1}^{1}\left(r^{2}+|v|^{2}-2 r|v| x\right)^{k / 2} r^{2} \mathrm{e}^{-\beta r^{2}} \mathrm{~d} x \mathrm{~d} r
$$

and, if $k=0, L(f)(v)=1$ for all $\dot{v} \in \mathbb{R}^{3}$. If $k=-1$, equation (2.1) reads

$$
\begin{aligned}
L(f)(v) & =4 \pi\left(\frac{\beta}{\pi}\right)^{3 / 2}\left[\int_{0}^{|v|} \frac{r^{2}}{|v|} \mathrm{e}^{-\beta r^{2}} \mathrm{~d} r+\int_{|v|}^{\infty} r \mathrm{e}^{-\beta r^{2}} \mathrm{~d} r\right] \\
& =\frac{\operatorname{erf}(\sqrt{\beta}|v|)}{|v|} .
\end{aligned}
$$

If $k=1$, equation (2.1) reads

$$
\begin{aligned}
L(f)(v) & =\frac{4 \pi}{3}\left(\frac{\beta}{\pi}\right)^{3 / 2}\left[\int_{0}^{|v|} \frac{r^{2}+3|v|^{2}}{|v|} r^{2} \mathrm{e}^{-\beta r^{2}} \mathrm{~d} r+\int_{|v|}^{\infty}\left(3 r^{2}+|v|^{2}\right) r \mathrm{e}^{-\beta r^{2}} \mathrm{~d} r\right] \\
& =\frac{1}{\sqrt{\beta \pi}} \mathrm{e}^{-\beta v^{2}}+\left(\frac{1}{2 \beta}+|v|^{2}\right) \frac{\operatorname{erf}(\sqrt{\beta}|v|)}{|v|} .
\end{aligned}
$$

Remark 2.2 Especially, if $v=0$,

$$
L(f)(0)= \begin{cases}1 & \text { if } k=0 \\ 2\left(\frac{\beta}{\pi}\right)^{1 / 2} & \text { if } k=-1 \\ \frac{2}{\sqrt{\beta \pi}} & \text { if } k=1\end{cases}
$$

For the truncated collision kernel $B^{\varepsilon}$ we get 
Proposition 2.3 Assume that $f$ is a (normalized) Maxwellian

$$
f(v)=\left(\frac{\beta}{\pi}\right)^{3 / 2} \mathrm{e}^{-\beta v^{2}}, \quad v \in \mathbb{R}^{3},
$$

and $\varepsilon>0$, then

$$
L^{\epsilon}(f)(v)=L(f)(v)+\Delta_{f}
$$

where

$$
\Delta_{f}= \begin{cases}2 \varepsilon\left(\frac{\beta}{\pi}\right)^{1 / 2} \mathrm{e}^{-\beta \varepsilon^{2}}-\operatorname{erf}(\sqrt{\beta \varepsilon}) & \text { if } k=0 \\ \frac{1}{|v|}\left(2 \varepsilon\left(\frac{\beta}{\pi}\right)^{1 / 2} \mathrm{e}^{-\beta \varepsilon^{2}}-\operatorname{erf}(\sqrt{\beta \varepsilon})\right) & \text { if } k=-1 \\ \frac{1}{|v|}\left[\left(\frac{\beta}{\pi}\right)^{1 / 2} \varepsilon\left(2|v|^{2}+\frac{2}{3} \varepsilon^{2}+\frac{1}{\beta}\right) \mathrm{e}^{-\beta \varepsilon^{2}}-\left(\frac{1}{2 \beta}+|v|^{2}\right) \operatorname{erf}(\sqrt{\beta \varepsilon})\right] & \text { if } k=1\end{cases}
$$

Proof

Using the same technique as given above, the results are obtained by changing the limits of the outer integral on the right hand side $(2.1)$ to the interval $[\delta, \infty]$.

Remark 2.4 The explicit formula given in Proposition 2.3 is used in the following subsection to show that the "beginning conditions" of the Kaniel-Shinbrot iteration scheme can be satisfied if the domain $\Omega$ is sufficiently small.

\subsection{Existence Theorem for Prescribed Boundary Values}

Consider for given $\varepsilon>0$ the boundary value problem

$$
v \cdot \nabla_{x} f+f L^{\varepsilon}(f)=Q_{+}^{\varepsilon}(f)
$$

with some instream conditions at the boundary $\partial \Omega$

$$
f(x, v)=\Phi(x, v), \quad x \in \partial \Omega, v \cdot n(x)>0 .
$$

We assume that we have upper and lower bounds on $\Phi$ of the type

$$
C_{1} \mathrm{e}^{-\gamma v^{2}} \leq \Phi(x, v) \leq C_{2} \mathrm{e}^{-\alpha v^{2}} .
$$

For simplicity we use in the following the notation $L=L^{\varepsilon}$ and $Q_{+}=Q_{+}^{\varepsilon}$.

Theorem 2.5 For any $\varepsilon>0, k=-1,0$ or 1 and $\Omega(\varepsilon)$ sufficiently small, there exists a solution of the boundary value problem (2.2)-(2.4).

Proof

We construct a solution via the Kaniel-Shinbrot iteration scheme [1]. Define two sequences $\left\{b_{n}\right\}_{n \in N}$ and $\left\{u_{n}\right\}_{n \in N}$ by

$$
\begin{aligned}
v \cdot \nabla_{x} l_{n+1}+l_{n+1} L\left(u_{n}\right) & =Q_{+}\left(l_{n}\right) \\
v \cdot \nabla_{x} u_{n+1}+u_{n+1} L\left(l_{n}\right) & =Q_{+}\left(u_{n}\right)
\end{aligned}
$$


together with boundary conditions

$$
l_{n+1}=u_{n+1}=\Phi, \quad x \in \partial \Omega, v \cdot n(x)>0
$$

and let $l_{0}=l_{0}(v)$. and $u_{0}=u_{0}(v)$ be two global Maxwellians with $l_{0}(v)<\Phi(x, v)<u_{0}(v)$ for all $x \in \partial \Omega$. A straightforward induction shows that the iteration defined by $(2.5)-(2.6)$ leads to monotone and bounded sequences in the form

$$
0 \leq l_{0} \leq l_{1} \leq l_{2} \leq \ldots \leq l_{n} \leq u_{n} \leq u_{n-1} \leq \ldots \leq u_{0}
$$

as long as we are able to choose $l_{0}$ and $u_{0}$ such that the "beginning condition"

$$
l_{0} \leq l_{1}
$$

and

$$
u_{1} \leq u_{0}
$$

will be satisfied. If $|v|<\varepsilon$ we simply get

$$
l_{n}(x, v)=\Phi(z(x, v), v)=u_{n}(x, v) \quad \forall n \in \mathbb{N}
$$

where

$$
z(x, v)=x-t v \in \partial \Omega, t=\inf _{\tau>0}\{\tau ; x-\tau v \in \partial \Omega\} .
$$

What remains is to investigate the case where $|v|>\varepsilon$.

The equations for $l_{1}$ and $u_{1}$ read

$$
\begin{aligned}
& v \cdot \nabla_{x} l_{1}+l_{1} L\left(u_{0}\right)=Q_{+}\left(l_{0}\right) \\
& v \cdot \nabla_{x} u_{1}+u_{1} L\left(l_{0}\right)=Q_{+}\left(u_{0}\right)
\end{aligned}
$$

Because we assume that $l_{0}$ and $u_{0}$ are (global) Maxwellians, we have

$$
\begin{aligned}
Q_{+}\left(l_{0}\right) & =l_{0} L\left(l_{0}\right) \\
Q_{+}\left(u_{0}\right) & =u_{0} L\left(u_{0}\right)
\end{aligned}
$$

Denote $L_{0}=L\left(l_{0}\right)$ and $U_{0}=L\left(u_{0}\right)$. Integrating equations (2.9) and (2.10) along a characteristic yields

$$
\begin{aligned}
l_{1}(x, v) & =\mathrm{e}^{-U_{0} s(x, v)} \Phi(x-s(x, v) v, v)+\left(1-\mathrm{e}^{-U_{0} s(x, v)}\right) \frac{L_{0} l_{0}}{U_{0}} \\
& =\frac{L_{0}}{U_{0}} l_{0}+\mathrm{e}^{-U_{0} s(x, v)}\left(\Phi(x-s(x, v) v, v)-\frac{L_{0}}{U_{0}} l_{0}\right)
\end{aligned}
$$

and

$$
\begin{aligned}
u_{1}(x, v) & =\mathrm{e}^{-L_{0} s(x, v)} \Phi(x-s(x, v) v, v)+\left(1-\mathrm{e}^{-L_{0} s(x, v)}\right) \frac{U_{0} u_{0}}{L_{0}} \\
& =\frac{U_{0}}{L_{0}} u_{0}+\mathrm{e}^{-L_{0} s(x, v)}\left(\Phi(x-s(x, v) v, v)-\frac{U_{0}}{L_{0}} u_{0}\right)
\end{aligned}
$$

with $L_{0}=L_{0}(v)$ and $U_{0}=U_{0}(v)$. 
Here $s(x, v)$ denotes the 'time' a particle with velocity $v$ needs to move from a boundary point $x-s(x, v) v$ to $x[4]$, i.e.

$$
s(x, v)=\inf _{\tau>0}\{\tau ; x-\tau v \in \partial \Omega, v \cdot n(x-\tau v)>0\} .
$$

Inequalities (2.7) and (2.8) will be satisfied if

$$
e^{-U_{0} s(x, v)} \geq \frac{U_{0}-L_{0}}{\frac{\Phi}{l_{0}} U_{0}-L_{0}}
$$

and

$$
\mathrm{e}^{-L_{0} s(x, v)} \geq \frac{U_{0}-L_{0}}{U_{0}-\frac{\Phi}{u_{0}} L_{0}},
$$

which should hold for all $z \in \bar{\Omega}$ and $v \in \mathbb{R}^{3}$ with $|v|>\varepsilon$.

The right hand side of (2.11) can be estimated by

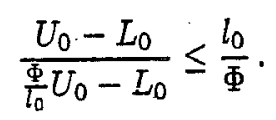

Hence, assuming that $l_{0}$ and $u_{0}$ are given by

$$
\begin{aligned}
u_{0} & =c_{1} \mathrm{e}^{-\gamma v^{2}} \\
u_{0} & =c_{2} \mathrm{e}^{-\alpha v^{2}}
\end{aligned}
$$

with $c_{1}<C_{1}$ and $c_{2}>C_{2}$ according to (2.4), we can choose, for a given $c_{2}, c_{1}$ sufficiently small to fulfill (2.11) as long as $s(x, v) U_{0}$ is uniformly bounded with respect to $x \in \Omega$ and $v \in \mathbb{R}^{3},|v|>\varepsilon$.

Equation (2.12) turns out to be more restrictive: using the estimates

$$
\mathrm{e}^{-L_{0} s(x, v)} \geq 1-L_{0} s(x, v)
$$

and

$$
\frac{U_{0}-L_{0}}{U_{0}-\frac{\Phi}{u_{0}} L_{0}} \leq 1+\left(\frac{\Phi}{u_{0}}-1\right) \frac{L_{0}}{U_{0}},
$$

we see that (2.12) will hold while

$$
s(x, v) \leq\left(1-\frac{\Phi}{u_{0}}\right) \frac{1}{U_{0}},
$$

which obviously restricts the size of $\Omega$. Because we use the truncated collision kernel as introduced in Section 2.1, we have

$$
s(x, v) \leq \frac{\operatorname{diam}(\Omega)}{\varepsilon} \quad \forall x \in \Omega, v \in \mathbb{R}^{3},|v|>\varepsilon
$$

where $\operatorname{diam}(\Omega)$ is the maximal distance between two boundary points. So we are able to satisfy the "beginning conditions" for $l_{0}$ and $u_{0}$, if

$$
\operatorname{diam}(\Omega) \leq\left(1-\frac{\Phi}{u_{0}}\right) \frac{\varepsilon}{U_{0}},
$$

where we still have to fix the constant $c_{2}$. 
For the following estimates we use the explicit formula for $L_{0}$ and $U_{0}$ as given by Proposition 2.3 to show that $s(x, v) U_{0}$ is uniformly bounded and equation (2.15) can be satisfied for a sufficiently small domain $\Omega$.

In the simplest case, i.e. $k=0$ (Maxwellian molecules), $L_{0}$ and $U_{0}$ are uniformly bounded for $x \in \Omega$ and $v \in \mathbb{R}^{3},|v|>\varepsilon$ by Proposition 2.3 .

Hence $s(x, v) U_{0}$ is uniformly bounded and the restriction on the domain $\Omega$ may read

$$
\operatorname{diam}(\Omega) \leq\left(\frac{\alpha}{\pi}\right)^{3 / 2} \frac{\varepsilon}{4 C_{2}}
$$

with $c_{2}=2 C_{2}$.

If $k=-1$, we estimate using Proposition (2.3)

$$
U_{0} \leq \frac{4}{\alpha} \pi c_{2}
$$

and therefore $s(x, v) U_{0}$ is uniformly bounded for $x \in \Omega$ and $v \in \mathbb{R}^{3},|v|>\varepsilon$. The restriction on $\Omega$ may read

$$
\operatorname{diam}(\Omega) \leq \frac{\alpha}{\pi} \frac{\varepsilon}{8 C_{2}}
$$

with $c_{2}=2 C_{2}$.

Finally, if $k=1$, we estimate

$$
s(x, v) U_{0} \leq c_{2} \frac{\operatorname{diam}(\Omega)}{\varepsilon}\left(M_{1}+\varepsilon M_{2}+\varepsilon^{2} M_{3}\right)
$$

where $M_{1}, M_{2}$ and $M_{3}$ are some constants depending only on $\alpha$ respectively $\gamma$. Furthermore we have

$$
s(x, v) \leq \frac{\operatorname{diam}(\Omega)}{|v|} \leq\left(1-\frac{\Phi}{u_{0}}\right) \frac{1}{U_{0}}
$$

which should hold for all $v \in \mathbb{R}^{3},|v|>\varepsilon$. Because $\frac{|v|}{U_{0}}$ is strictly positive and monotonically increasing with $|v|$, we get

$$
\operatorname{diam}(\Omega) \leq\left(\frac{\alpha}{\pi}\right)^{3 / 2} \frac{\varepsilon}{2 U_{0}}
$$

with $c_{2}=2 C_{2}$.

The two sequences $\left\{l_{n}\right\}_{n \in N}$ and $\left\{u_{n}\right\}_{n \in N}$ are monotone and bounded and therefore convergent. If we assume, that

$$
l=\lim _{n \rightarrow \infty} l_{n}
$$

and

$$
u=\lim _{n \rightarrow \infty} u_{n}
$$

we find that

$$
\begin{aligned}
& v \cdot \nabla_{x} l+l L(u)=Q_{+}(l) \\
& v \cdot \nabla_{x} u+u L(l)=Q_{+}(u)
\end{aligned}
$$

together with the boundary condition

$$
u-l=0, \quad x \in \partial \Omega, v \cdot n(x)>0 .
$$


It remains to show that $l=u$.

Define $h(x, v)=u(x, v)-l(x, v)$. Then the equation for $h$ reads

$$
v \cdot \nabla h+h L(l)-l L(h)=Q_{+}(u)-Q_{+}(l) .
$$

Integrating over $\mathbb{R}^{3}$ and the domain $\Omega$ gives

$$
\iint_{\Omega} v \cdot \nabla h \mathrm{~d} v \mathrm{~d} x=\iint_{\mathbb{R}^{3}}\left(Q_{\boldsymbol{R}^{3}}(u)-Q_{+}(l)+l L(h)-h L(l)\right) \mathrm{d} v \mathrm{~d} x .
$$

Because

$$
\int_{\Omega} \int_{R^{3}}\left(Q_{+}(u)-Q_{+}(l)\right) \mathrm{d} v \mathrm{~d} x=\iint_{\Omega}(u L(u)-l L(l)) \mathrm{d} v \mathrm{~d} x
$$

we get

$$
\int_{\Omega} \int_{\boldsymbol{R}^{3}} v \cdot \nabla h \mathrm{~d} v \mathrm{~d} x=\int_{\Omega} \int_{\boldsymbol{R}^{3}}(u+l) L(h) \mathrm{d} v \mathrm{~d} x .
$$

Applying the divergence theorem yields

$$
-\int_{\partial \Omega} \int_{v \cdot n<0}|v \cdot n| h(x, v) \mathrm{d} v \mathrm{~d} \sigma(x)=\iint_{\Omega \cdot \mathbb{R}^{3}}(u+l) L(h) \mathrm{d} v \mathrm{~d} x .
$$

Because $h(x, v) \geq 0$ and therefore $L(h)(x, v) \geq 0$, we get that $h(x, v)=0$ a.e. on $\Omega \times \mathbb{R}^{3}$, which completes the proof.

Remark 2.6 The restrictions on the size of the domain $\Omega$ depend - by construction - strongly on the truncation parameter $\varepsilon$. There is also a strong dependence on the given boundary flux $\Phi$. If $\Phi$ is sufficiently small, i.e., if the gas conditions are near to a vacuum, the restrictions on $\Omega$ become weaker.

Within the given upper and lower bounds the solution of Theorem 2.5 is unique.

Theorem 2.7 Suppose $\varepsilon>0$ given and $g$ is a solution of (2.2)-(2.4) with

$$
l_{0}(v) \leq g(x, v) \leq u_{0}(v) \quad \forall x \in \Omega, v \in \mathbb{R}^{3},
$$

where $l_{0}$ and $u_{0}$ are the lower and upper bounds on the solution $f$ given by Theorem 2.5. Then $f=g$ almost everywhere.

Proof

Because $g$ is a solution, we have $v \cdot \nabla_{x} g=Q_{+}(g)-g L(g)$. Suppose that $l_{n}$ and $u_{n}$ are the $n$-th iterations as formulated in Theorem 2.5. Then

$$
\begin{aligned}
v \cdot \nabla_{x}\left(g-l_{n}\right)+\left(g-l_{n}\right) L\left(u_{n-1}\right) & =g L\left(u_{n-1}-g\right)+Q_{+}(g)-Q_{+}\left(l_{n-1}\right) \\
v \cdot \nabla_{x}\left(u_{n}-g\right)+\left(u_{n}-g\right) L\left(l_{n-1}\right) & =g L\left(g-l_{n-1}\right)+Q_{+}\left(u_{n-1}\right)-Q_{+}(g)
\end{aligned}
$$

and with $l_{0} \leq g \leq u_{0}$ we get inductively

$$
l_{n} \leq g \leq u_{n} \quad \forall n \in \mathbb{N} .
$$

By Theorem 2.5 both sequences $\left\{l_{n}\right\}_{n \in N}$ and $\left\{u_{n}\right\}_{n \in N}$ are convergent with

$$
\lim _{n \rightarrow \infty} l_{n}=f=\lim _{n \rightarrow \infty} u_{n}
$$

hence $f=g$ almost everywhere. 


\subsection{Existence Theorem for Diffusive Boundary Conditions}

For diffusive boundary conditions we consider for any $\varepsilon>0$ the steady Boltzmann equation (2.2) together with the boundary condition

$$
f(x, v)=m_{\beta(x)}(v) \int_{v_{*} \cdot n<0}\left|v_{*} \cdot n\right| f\left(x, v_{*}\right) \mathrm{d} v_{*} \quad \forall x \in \partial \Omega, v \cdot n>0
$$

where

$$
m_{\beta(x)}(v)=\frac{2 \beta^{2}(x)}{\pi} \mathrm{e}^{-\beta(x) v^{2}}
$$

and $\alpha \leq \beta(x) \leq \gamma \quad \forall x \in \partial \Omega$.

The diffusive boundary conditions are more difficult to handle. Obviously, $f \equiv 0$ is a trivial solution of the problem. The homogeneity of the boundary condition suggest that a free parameter enters the problem, e.g., the mass $M=\iint_{\Omega} f(x, v) \mathrm{d} v \mathrm{~d} x$. More about the choice of a free parameter can be found in Section 3 .

Remark 2.8 For the free transport equation, i.e. equation (2.2) without collision integral, the diffusive boundary conditions were already studied in [4].

In the following we try to prove an existence result based on the theorems presented in Subsection 2.2. The idea is to investigate the so-called Albedo operator $\mathcal{A}$ which connects the in-and outgoing fluxes at the boundary $\partial \Omega$.

With the result of Section 2.2 we are able to obtain a solution $f(x, v)$ of the steady Boltzmann equation (2.2) by prescribing the ingoing flux $\Phi(x, v)$. Hence the.Albedo operator $\mathcal{A}$ is well defined: suppose that $\Phi(x, v)$ is given for $x \in \partial \Omega$ and $v \in \mathbb{R}^{3}$ such that $v \cdot n(x)>0$. Then we have a solution $f(x, v)$ on $\bar{\Omega} \times \mathbb{R}^{3}$ with

$$
f(x, v)=\Phi(x, v), \quad x \in \partial \Omega, v \cdot n(x)>0 .
$$

The outgoing flux $f(x, v)$ at the boundary $\partial \Omega$ exactly defines the Albedo operator $\mathcal{A}$,

$$
\mathcal{A}[\Phi](x, v)=f(x, v), \quad x \in \partial \Omega, v \cdot n(x)<0
$$

Now we are able to transform problem (2.2) with boundary condition (2.16) into a fixed point problem: given the (inverse) temperature profile $\beta(x)$ on the boundary $\partial \Omega$ we are looking for a fixed point of the operator $\mathcal{R}$ defined by

$$
(\mathcal{R} \Phi)(x, v)=m_{\beta(x)}(v) \int_{v_{0} \cdot n(x)<0}\left|v_{*} \cdot n\right| \mathcal{A}[\Phi]\left(x, v_{*}\right) \mathrm{d} v_{*}
$$

for $x \in \partial \Omega$ and $v \cdot n(x)>0$.

Remark 2.9 Due to the bounds on the solution $f(x, v)$, the operator $\mathcal{R}$ is well defined.

If a fixed point $\Phi$ of $\mathcal{R}$ exists, then using the results of Section 2.2 we get a solution of (2.2) which fulfills the diffusive boundary conditions formulated in (2.16).

For simplicity we first consider the simpler problem of a free transport equation

$$
v \cdot \nabla_{x} f=0
$$


with boundary condition $f(x, v)=\Phi(x, v), \quad x \in \partial \Omega, v \cdot n(x)>0$.

The solution is given by

$$
f(x, v)=\Phi(z(x, v), v)
$$

where $z(x, v)$ is the corresponding boundary point for $x \in \Omega$ following the characteristic line $\{x-s v, s>0\}$.

Remark 2.10 Obviously (2.19) holds for arbitrary bounded domains $\Omega$ without any restriction on the size of $\Omega$ as discussed in the previous section.

Moreover, $\mathcal{A}$ is a linear operator with

$$
\mathcal{A}[\Phi](x, v)=\Phi(z(x, v), v)
$$

for $x \in \Omega$ and $v \cdot n(x)<0$.

Now consider equation (2.18) together with the diffusive boundary condition (2.16). We are then looking for a fixed point of the operator $\mathcal{R}$ defined by

$$
(\mathcal{R} \Phi)(x, v)=m_{\beta(x)}(v) \int_{v_{*} \cdot n<0}\left|v_{*} \cdot n\right| \Phi\left(z\left(x, v_{*}\right), v_{*}\right) \mathrm{d} v_{*}
$$

Because $\mathcal{A}$ is linear, one fixed point $\Phi$ of (2.20) directly leads to infinitely many fixed points by multiplying $\Phi$ with a non-negative constant $c$. Furthermore we have

Theorem 2.11 Let $\Phi$ be a global Maxwellian

$$
\Phi(x, v)=c \mathrm{e}^{-\alpha v^{2}}, \quad x \in \Omega, v \in \mathbb{R}^{3}
$$

and $\beta(x), x \in \partial \Omega$, a given (inverse) temperature profile along the boundary $\partial \Omega$. Then $\mathcal{R}^{2} \Phi=\mathcal{R} \Phi$ and $\mathcal{R} \Phi$ is a fixed point of $\mathcal{R}$.

Proof

If $\Phi$ is a global Maxwellian

$$
(\mathcal{R} \Phi)(x, v)=\frac{c \pi}{2 \alpha^{2}} m_{\beta(x)}=\frac{c}{\alpha^{2}} \beta^{2}(x) \mathrm{e}^{-\beta(x) v^{2}}
$$

and

$$
\mathcal{R}(\mathcal{R} \Phi)(x, v)=m_{\beta(x)} \int_{v_{*} \cdot n<0}\left|v_{*} \cdot n\right| \mathcal{A}[\mathcal{R} \Phi]\left(x, v_{*}\right) \mathrm{d} v_{*}
$$

Using (2.21) yields

$$
\mathcal{R}(\mathcal{R} \Phi)(x, v)=\frac{c}{\alpha^{2}} m_{\beta(x)} \int_{v_{*} \cdot n_{<0}}\left|v_{*} \cdot n\right| \beta^{2}\left(z\left(x, v_{*}\right)\right) \mathrm{e}^{-\beta\left(z\left(x, v_{*}\right)\right) v_{*}^{2}} \mathrm{~d} v_{*} .
$$

The right hand side of $(2.22)$ can be written as [4]

$$
\int_{v_{*} \cdot n<0}\left|v_{*} \cdot n\right| \beta^{2} \mathrm{e}^{-\beta v_{*}^{2}} \mathrm{~d} v_{*}=\int_{e \cdot n,|e|=1}|e \cdot n| \int_{0}^{\infty}\left|v_{*}\right|^{3} \beta^{2}(y) \mathrm{e}^{-\beta(y)\left|v_{-}\right|^{2}} \mathrm{~d}\left|v_{*}\right| \mathrm{d} e,
$$


where $y$ is the corresponding boundary point of $x \in \partial \Omega$ in direction $e$.

Substituting $\tau=\sqrt{\beta}\left|v_{*}\right|$ and calculating the remaining integrals gives

$$
\int_{v_{*} \cdot n<0}\left|v_{*} \cdot n\right| \beta^{2} \mathrm{e}^{-\beta v_{*}^{2}} \mathrm{~d} v_{*}=\frac{\pi}{2}
$$

Hence,

$$
\mathcal{R}(\mathcal{R} \Phi)(x, v)=\frac{c}{\alpha^{2}} \beta^{2}(x) \mathrm{e}^{-\beta(x) v^{2}}=(\mathcal{R} \Phi)(x, v)
$$

and $\mathcal{R} \Phi$ is a fixed point of $\mathcal{R}$, which completes the proof.

The Albedo aperator $\mathcal{A}$ of the Boltzmann equation is obviously nonlinear. However, if $\Phi$ is a fixed point of $\mathcal{R}$, then, multiplying $\Phi$ with an arbitrary constant $c \in \mathbb{R}_{+}, c \Phi$ is a fixed point of the equation

$$
v \cdot \nabla_{x} f=\frac{1}{c} Q(f, f)
$$

together with boundary condition (2.16).

Because of the nonlinearity of $\mathcal{A}$, the existence of a fixed point of $(2.20)$ is nontrivial. For a given flux $\Phi$, let

$$
j(x)=\int_{v \cdot n<0}|v \cdot n| \mathcal{A}[\Phi](x, v) \mathrm{d} v
$$

along the boundary $\partial \Omega$. Assuming that $\Phi$ is a fixed point of $(2.20), \Phi$ must be of the form

$$
\Phi(x, v)=j(x) m_{\beta(x)}(v), \quad x \in \partial \Omega, v \cdot n(x)>0 .
$$

Hence, we are looking for a function $j(x)$ such that

$$
j(x)=(\mathcal{R} j)(x)=\int_{v \cdot n<0}|v \cdot n| \mathcal{A}\left[j m_{\beta}\right](x, v) \mathrm{d} v
$$

Remark 2.12 For the free transport equation the fixed points of (2.23) are given by the constant functions, i.e. $j(x)=c \in \mathbb{R}_{+}$.

Lemma 2.13 Let $j \in L^{1}(\partial \Omega)$ then

$$
\int_{\partial \Omega}(\mathcal{R} j)(x) \mathrm{d} \sigma(x)=\int_{\partial \Omega} j(x) \mathrm{d} \sigma(x) .
$$

Proof

Consider the problem

$$
v \cdot \nabla_{x} f=Q(f, f)
$$

with boundary condition $f(x, v)=j(x) m_{\beta(x)}(v)$ for all $x \in \partial \Omega, v \cdot n(x)>0$. Integrating (2.24) with respect to $x$ and $v$ and applying the divergence theorem yields

$$
\int_{\partial \Omega} \int_{v \cdot n>0}|v \cdot n| j(x) m_{\beta(x)}(v) \mathrm{d} v \mathrm{~d} \sigma(x)=\int_{\partial \Omega} \int_{v \cdot n<0}|v \cdot n| f(x, v) \mathrm{d} v \mathrm{~d} \sigma(x) .
$$


Because of mass conservation at $\partial \Omega$, we have

$$
\int_{\partial \Omega} \int_{v \cdot n>0}|v \cdot n| j(x) m_{\beta(x)}(v) \mathrm{d} v \mathrm{~d} \sigma(x)=\int_{\partial \Omega} j(x) \mathrm{d} \sigma(x) .
$$

Hence, equation (2.25) reads

$$
\begin{aligned}
\int_{\partial \Omega} j(x) \mathrm{d} \sigma(x) & =\int_{\partial \Omega} \int_{v \cdot n<0}|v \cdot n| \mathcal{A}\left[j m_{\beta}\right](x, v) \mathrm{d} v \mathrm{~d} \sigma(x) \\
& =\int_{\partial \Omega}(\mathcal{R} j)(x) \mathrm{d} \sigma(x) .
\end{aligned}
$$

The energy flux of $\mathcal{A}\left[j m_{\beta}\right](x, v)$ can be estimated by

Lemma 2.14 Let $j \in L^{1}(\partial \Omega)$ and $\beta(x)$ be a bounded (inverse) temperature profile along $\partial \Omega$. Then

$$
\int_{\partial \Omega} \int_{v \cdot n<0}|v \cdot n| v^{2} \mathcal{A}\left[j m_{\beta}\right](x, v) \mathrm{d} v \mathrm{~d} \sigma(x)=2 \int_{\partial \Omega} \beta(x) j(x) \mathrm{d} \sigma(x) .
$$

\section{Proof}

Multiplying (2.24) by $v^{2}$ and integrating with respect to $x$ and $v$ yields

$$
\int_{\Omega} \int_{R^{3}} v v^{2} \nabla_{x} f \mathrm{~d} x \mathrm{~d} v=0 .
$$

Applying the divergence theorem equation (2.26) reads

$$
\int_{\partial \Omega} \int_{v \cdot n>0}|v \cdot n| v^{2} j(x) m_{\beta(x)}(v) \mathrm{d} v \mathrm{~d} \sigma(x)-\int_{\partial \Omega} \int_{v \cdot n<0}|v \cdot n| v^{2} \mathcal{A}\left[j m_{\beta}\right](x, v) \mathrm{d} v \mathrm{~d} \sigma(x)=0 .
$$

Finally,

$$
\int_{\partial \Omega} \int_{v \cdot n>0}|v \cdot n| v^{2} j(x) m_{\beta(x)}(v) \mathrm{d} v \mathrm{~d} \sigma(x)=2 \int_{\partial \Omega} \beta(x) j(x) \mathrm{d} \sigma(x) .
$$

In the one-dimensional case, $\Omega$ is an interval $[0, a], a>0$ on $\mathbb{R}$, we have exactly two boundary points and the lemma above reads

$$
(\mathcal{R} j)(0)+(\mathcal{R} j)(a)=j(0)+j(a) .
$$

Because all terms on (2.27) are non-negative, $\mathcal{R}$ is a mapping from $L_{c}=\left\{(x, y) \in \mathbb{R}^{2}, x+y=j\right\}$ into itself, where $j$ is an arbitrary positive constant describing the amount of mass flux into the intervall $[0, a]$.

If $a$ is small enough (with respect to $j$ ) to apply the existence theorem of Section 2.2 , we automatically get the existence of a fixed point, because $\mathcal{R}: L_{c} \rightarrow L_{c}$ is a continuous mapping.

Theorem 2.I5 On a sufficiently small intervall $[0, a] \subset \mathbb{R}$ there exists a solution of the boundary value problem (2.2) with diffusive boundary conditions (2.16).

The generalization to multidimensions remains an open question - due to the lack of local estimates on $(\mathcal{R} j)$. 


\section{Global Existence Results in the One-Dimensional Case}

Global existence results for the steady one-dimensional slab problem were given in Ref. [3] for discrete-velocity models and in Ref. [2] for the full Boltzmann equation. The boundary conditions were either prescribed fluxes at both sides - used in [2] and [3] - or prescribed flux at one side and diffusive conditions on the other side [3]. In the following two sections it is shown how to generalize these results to the case of purely diffusive boundary conditions.

\subsection{Discrete-Velocity Models}

We recall the global existence result for discrete-velocity models as formulated in [3]. The discretevelocity model in one space dimension is given by the set of ordinary differential equations

$$
\xi_{i} \frac{\mathrm{d} f^{i}}{\mathrm{~d} x}=Q^{i}(f), \quad i=1, \ldots, n,
$$

where $f=\left(f^{1}, \ldots, f^{n}\right)$ are the particle densities associated with the $\mathrm{n}$ admissible velocities $u_{i} \in \mathbb{R}^{3}$ and $\xi_{i}$ are the $x$-components of the vectors $u_{i}$. Each $Q^{i}$ has the form

$$
Q^{i}(f)=\sum_{j, k, l} A_{k l}^{i j}\left(f^{k} f^{l}-f^{i} f^{j}\right)
$$

such that conservation of mass, momentum and energy is fulfilled.

Remark 3.1 The result given in [3] even holds for more general types of collision terms $Q^{i}$. For example, conservation of energy is not required.

We consider (3.1) in the slab $0<x<a$ under the additional assumption that $\xi_{i} \neq 0$ for all $i=1, \ldots, n$. In [3] it was shown that (3.1) together with the boundary conditions

$$
\begin{aligned}
& f^{i}(0)=\alpha^{i}, \quad \xi_{i}>0, \quad\left(\alpha^{i} \geq 0\right) \\
& f^{i}(a)=\beta^{i} \sum^{+} \xi_{j} f^{j}(a), \quad \xi_{i}<0, \quad\left(\beta^{i} \geq 0\right)
\end{aligned}
$$

has a global solution for arbitrary slab length $a$. Here, $\Sigma^{+}\left(\Sigma^{-}\right)$means that the sum is taken over all positive (negative) velocities $\xi_{j}$ and the coefficients $\beta_{i}$ are normalized such that $\sum^{-} \xi_{i} \beta^{i}=-1$.

Theorem $3.2[3]$

The problem (3.1) with boundary conditions (3.2),(3.3) has a solution in $\left[\mathrm{C}_{+}^{0}\right]^{\text {n }}$.

$\mathcal{C}_{+}^{0}$ denotes the nonnegative, continuous functions in the interval $[0, a]$ and $\left[\mathcal{C}_{+}^{0}\right]^{n}$ is the Cartesian product of $n$ copies of $\mathcal{C}_{+}^{0}$.

The boundary conditions formulated in (3.2) and (3.3) are not exactly diffusive boundary conditions as given in Subsection 2.3 because the ingoing flux on the left hand side of the slab is prescribed. Complete diffusive boundary conditions will be of the form

$$
\begin{array}{ll}
f^{i}(0)=\beta_{0}^{i} \sum^{-} \xi_{j} f^{j}(0), & \xi_{i}>0 \\
f^{i}(a)=\beta_{0}^{i} \Sigma^{+} \xi_{j} f^{j}(a), & \xi_{i}<0
\end{array}
$$

with $\sum^{+} \xi_{i} \beta_{0}^{i}=1$ and $\sum^{-} \xi_{i} \beta_{a}^{i}=-1$.

However, the result formulated in [3] can be used to show the existence of solutions of problem (3.1) together with the purely diffusive boundary conditions (3.4) and (3.5). As the boundary 
conditions (3.4), (3.5) are homogeneous in $f$, we expect a family of solutions (note that $f \equiv 0$ is a solution). It turns out that the outgoing flux $j^{-}(0)=\Sigma^{-}\left|\xi_{j}\right| f^{j}(0)$ can be chosen as a free parameter.

Denote by $j(x)$ the mass flux inside the slab, i.e. $j(x)=\sum \xi_{i} f^{i}(x), j^{+}(x)=\sum^{+} \xi_{i} f^{i}(x)$ and $j^{-}(x)=\sum^{-}\left|\xi_{i}\right| f^{i}(x)$. Because of mass conservation, every solution of (3.1) satisfies

$$
\frac{\mathrm{d} j(x)}{\mathrm{d} x}=0
$$

and with the boundary condition (3.3) we have $j(x)=0$ for all $0 \leq x \leq a$.

Now consider equation (3.1) with boundary conditions

$$
\begin{aligned}
& f^{i}(0)=\beta_{0}^{i} \tilde{j}^{-}(0), \quad \xi_{i}>0 \\
& f^{i}(a)=\beta_{a}^{i} \Sigma^{+} \xi_{j} f^{j}(a), \quad \xi_{i}<0,
\end{aligned}
$$

where $\bar{j}^{-}(0)$ is some given positive constant, $\sum^{+} \xi_{i} \beta_{0}^{i}=1$ and $\Sigma^{-} \xi_{i} \beta_{a}^{i}=-1$. Due to the result of [3] there exists a solution and

$$
\frac{\mathrm{d} j(x)}{\mathrm{d} x}=0, \quad j(a)=0 .
$$

Hence $j(0)=0$ and $j^{-}(0)=\tilde{j}^{-}(0)$, so we can state the following result.

\section{Theorem 3.3}

The problem (3.1) with boundary conditions (3.4), (3.5) has a one parameter family of solutions in $\left[\mathcal{C}_{+}^{0}\right]^{n}$. The outgoing flux $j^{-}(0)$ parametrizes these solutions.

\section{Proof}

Consider equation (3.1) with boundary conditions (3.6); (3.7). By Theorem 3.2 the problem has a solution in $\left[C_{+}^{0}\right]^{n}$. Because of $j^{-}(0)=\bar{j}^{-}(0)$, the solution also fulfills the boundary conditions (3.4), (3.5).

\subsection{Measure Solutions in a Slab}

In this section we consider the steady Boltzmann equation in a slab $0 \leq x \leq a$

$$
\xi \frac{\mathrm{d}}{\mathrm{d} x} f=Q(f),
$$

where $\xi$ denotes the $x$-component of the velocity $v$. Our final goal will be to show the existence of a solution of (3.8) together with the diffusive boundary conditions

$$
\begin{array}{ll}
f(0, v)=j^{-}(0) m_{0}(v), & \xi>0 \\
f(a, v)=j^{+}(a) m_{a}(v), & \xi<0
\end{array}
$$

and $j^{-}(0)=\int_{\xi<0}|\xi| f(0, v) \mathrm{d} v, j^{+}(a)=\int_{\xi>0}|\xi| f(a, v) \mathrm{d} v$. Here, $m_{0}(v)$ and $m_{a}(v)$ are two (normalized) half-space Maxwellians,

$$
m_{0}(v)=\frac{2 \beta_{0}^{2}}{\pi} \mathrm{e}^{-\beta_{0} v^{2}} \quad \text { and } \quad m_{a}(v)=\frac{2 \beta_{a}^{2}}{\pi} \mathrm{e}^{-\beta_{a} v^{2}} .
$$

In [2] an existence result for problem (3.8) together with the boundary conditions

$$
\begin{aligned}
& f(0, v)=f_{0}(v) \\
& f(a, v)=f_{a}(v)
\end{aligned}
$$


was given. The solution was found in the space of measure-valued functions of $x$,

$$
x \rightarrow \mu_{x}, \quad[0, a] \rightarrow M,
$$

where $M$ denotes the set of bounded measures on $\mathbb{R}^{3}$. We outline the strategy followed in Ref. [2], without giving all the technical details.

The single steps to the existence result are the following: first of all one passes to the measure formulation of problem (3.10) which yields the equation

$$
\xi \frac{\mathrm{d}}{\mathrm{d} x} \mu_{x}=Q\left(\mu_{x}, \mu_{x}\right)
$$

in the sense of weak*-convergence of measures. The collision kernel is assumed to be of the form (1.2) with $-1 \leq k \leq 0$. The kernel is first truncated in the same way as in Subsection 2.1 and further by a crude truncation of the form

$$
B^{\delta}=B_{\varepsilon} k_{\delta}
$$

with

$$
k_{\delta}= \begin{cases}1 & \text { if } v^{2}+v_{*}^{2} \leq \delta^{-2}, \min \left\{|\xi|,\left|\xi_{*}\right|,\left|\xi^{\prime}\right|,\left|\xi_{*}^{\prime}\right|\right\}>\delta \text { or }\left|v-v_{*}\right|>\delta \\ 0 & \text { otherwise }\end{cases}
$$

so that equation (3.14) is replaced by

$$
\xi \frac{\mathrm{d}}{\mathrm{d} x} \mu_{x}=Q^{\delta}\left(\mu_{x}, \mu_{x}\right)
$$

with boundary conditions $\left.\mu_{0}\right|_{\{\xi>0\}}=\mu_{0}^{+}$and $\left.\mu_{a}\right|_{\{\xi<0\}}=\mu_{a}^{-}$. In the following we will use the notation $\|\mu \cdot\|=\sup _{x \in[0, a]} \int \mathrm{d} \mu_{x}(v)$.

The collision operator $Q^{\delta}\left(\mu_{x}, \mu_{x}\right)$ can be written in the form

$$
Q^{\delta}\left(\mu_{x}, \mu_{x}\right)=Q_{+}^{\delta}\left(\mu_{x}, \mu_{x}\right)-L^{\delta}\left(\mu_{x}\right) \mu_{x},
$$

where $Q_{+}^{\delta}\left(\mu_{x}, \mu_{x}\right), L^{\delta}\left(\mu_{x}\right) \mu_{x}$ are measures defined by

$$
\left\langle Q_{+}^{\delta}\left(\mu_{x}, \mu_{x}\right), \varphi\right\rangle=\iint_{v} \int_{v_{*}} B^{\delta}\left(v, n, v_{*}\right) \varphi(v) \mathrm{d}\left(M_{x} \circ J\right)
$$

with $\mathrm{d} M_{x}=\mathrm{d} \omega(n) \mathrm{d} \mu_{x} \times \mathrm{d} \mu_{x}$ and

$$
\left\langle L^{\delta}\left(\mu_{x}\right) \mu_{x}, \varphi\right\rangle=\left\langle\mu_{x}, L^{\delta}\left(\mu_{x}\right) \varphi\right\rangle .
$$

Because of the truncation of $B$, we have

$$
\left\|L^{\delta}\left(\mu_{x}\right)(x)\right\| \leq 4 \pi C(\delta) \int \mathrm{d} \mu_{x}(w)
$$

with $B^{\delta} \leq C(\delta)$.

Let $\bar{X}=(\mathcal{C}[0, a] ; M)$ be the cone of all continuous functions $[0, a] \rightarrow M$ and $B_{R}(0) \subset X$ the set of all continuous measure-valued functions $\mu$. such that $\| \mu$. $\| \leq R$. For $\tau \geq 4 \pi C(\delta)$ one studies the operators

$$
T(\tau): B_{R}(0) \rightarrow X, \quad v .=T(\tau) \mu
$$


defined by

$$
\begin{gathered}
\left.\nu_{0}\right|_{\{\xi>0\}}=\mu_{0}^{+},\left.\quad \nu_{a}\right|_{\{\xi<0\}}=\mu_{a}^{-}, \\
\xi \frac{\mathrm{d}}{\mathrm{d} x} \nu_{x}=0
\end{gathered}
$$

for $|\xi| \leq \delta,|\xi| \geq 1 / \delta$ and

$$
\xi \frac{\mathrm{d}}{\mathrm{d} x} \nu_{x}+\tau \rho[\mu .] .(x) \nu_{x}=Q_{+}^{\delta}\left(\mu_{x}, \mu_{x}\right)+\tau \rho[\mu .](x) \mu_{x}-L^{\delta}\left(\mu_{x}\right) \mu_{x}
$$

for $\delta<|\xi|<1 / \delta, \rho[\mu]=.\int \mathrm{d} \mu .(v)$.

The boundary value problem (3.17)-(3.19) has a unique solution $\nu$, and the mapping $\mu . \rightarrow \nu$ : is continuous from $B_{R}(0)$ into $X$. Because $T(\tau)$ will in general not map $B_{R}(0)$ into itself, one introduces the retract $T_{R}: X \rightarrow B_{R}(0)$, defined by

$$
\left(T_{R} \mu .\right) .= \begin{cases}\mu . & \text { if }\|\mu .\| \leq R \\ \frac{R}{\|\mu .\|} \mu . & \text { if }\|\mu .\|>R\end{cases}
$$

Now $T_{R} \circ T(\tau) B_{R}(0)$ is relatively compact and has a fixed point in $B_{R}(0)$. To proof the existence of a fixed point of $T(\tau)$ in some $B_{R}(0)$ it remains to show that the set of all fixed points of $T_{R} \circ T(\tau)$ is uniformly bounded. This was achieved using the conservation quantities of the Boltzmann equation. Finally it was shown - using the usual Cantor diagonalization - how to get rid of the crude truncation.

In order to handle diffusive boundary conditions (3.9), (3.10) we first generalize the existence result given above to the case where the boundary condition (3.13) is replaced by general stochastic scattering conditions of the form

$$
|\xi| f(a, v)=\int_{\xi^{\prime}>0} R_{a}\left(v^{\prime} \rightarrow v\right)\left|\xi^{\prime}\right| f(a, v) \mathrm{d} v
$$

with an appropiate boundary kernel $R_{a}$. In a second step we show - similar to the consideration in the previous section - how to extract the case of purely diffusive boundary conditions (3.9), (3.10).

As in [2] we consider the measure formulation of problem (3.8) together with the boundary conditions

$$
\begin{aligned}
& \left.\mu_{0}\right|_{\{\xi>0\}}=\mu_{0}^{+} \\
& \left.\mu_{a}\right|_{\{\xi<0\}}=\int_{\xi^{\prime}>0} R_{a}\left(v^{\prime} \rightarrow v\right) \frac{\left|\xi^{\prime}\right|}{|\xi|} \mathrm{d} \mu_{a}(v)
\end{aligned}
$$

Here, the boundary kernel $R_{a}\left(v^{\prime} \rightarrow v\right)$ should fulfll the following conditions [5]

$$
\begin{gathered}
R \geq 0, \\
\int_{\xi<0} R_{a}\left(v^{\prime} \rightarrow v\right) \mathrm{d} v=1
\end{gathered}
$$

(mass conservation),

$$
\exists c_{1}>0 \text { such that } \int_{\xi<0} R_{a}\left(v^{\prime} \rightarrow v\right)|\xi| \mathrm{d} v \geq c_{1}
$$


("spreading condition") and

$$
\exists c_{2}>0 \text { such that } \int_{\xi<0} R_{a}\left(v^{\prime} \rightarrow v\right) v^{2} \mathrm{~d} v \leq c_{2}
$$

("energy condition").

Using the same truncation $B^{\delta}$ of the collision kernel as above, we define the operator $T(\tau)$ as in (3.16) where the boundary conditions (3.17) are replaced by (3.21). Following the same analysis as in [2] we are able to prove the existence of a fixed point of $T_{R} \circ T(\tau) B_{R}(0)$ where $T_{R}$ is the retract defined in (3.20). As mentioned above it remains to prove that the set of all fixed points of $T_{R} \circ T(\tau)$ is uniformly bounded. This can be done following similar arguments as in [2]:

Lemma 3.4 For any solution $\mu^{T}$ of the equation

$$
T_{R} \circ T(\tau) \mu^{\tau}=\mu^{\tau}
$$

with boundary conditions (3.21) we have

$$
\max _{x \in[0, a]} \int \xi^{2} \mathrm{~d} \mu_{x}^{\tau}(v) \leq C\left(\mu_{0}^{+}\right)
$$

Proof

Let $j^{+}(x)=\int_{\xi>0} \xi \mathrm{d} \mu_{x}(v), j^{-}(x)=\int_{\xi<0}|\xi| \mathrm{d} \mu_{x}(v), j=j^{+}-j^{-}$and $p^{+}(x)=\int_{\xi>0} \xi^{2} \mathrm{~d} \mu_{x}(v), p^{-}(x)=$ $\int_{\xi<0} \xi^{2} \mathrm{~d} \mu_{x}(v), p=p^{+}+p^{-}$, then by the usual conservation laws,

$$
\int \varphi(v) \mathrm{d}\left[Q_{+}^{\delta}\left(\mu_{x}, \mu_{x}\right)-L^{\delta}\left(\mu_{x}\right) \mu_{x}\right](v)=0,
$$

where $\varphi(v)=1, v$ or $v^{2}$. Hence, if $\mu^{\tau}$. is a fixed point of $T_{R} \circ T(\tau)$,

$$
\frac{\mathrm{d} j}{\mathrm{~d} x}=(\lambda-1) \tau \rho^{2}
$$

and

$$
\frac{\mathrm{d} p}{\mathrm{~d} x}=(\lambda-1) \tau \rho j
$$

where $\lambda=\min \left\{\frac{R}{\| T(\tau) \mu ? \pi}, 1\right\}$.

Because $\rho(x) \geq 0$, we have $j(x)=j^{+}(x)-j^{-}(x) \leq j^{+}(0)-j^{-}(0)$ and

$$
\begin{aligned}
j(a) & =j^{+}(a)-j^{-}(a) \\
& =\int_{\xi>0} \xi \mathrm{d} \mu_{x}(v)-\int_{\xi<0}|\xi| \mathrm{d} \mu_{x}(v) \\
& =\int_{\xi>0} \xi \mathrm{d} \mu_{x}(v)-\int_{\xi<0} \int_{\xi^{\prime}>0} R_{a}\left(v^{\prime} \rightarrow v\right)\left|\xi^{\prime}\right| \mathrm{d} \mu_{x}\left(v^{\prime}\right) \mathrm{d} v \\
& =0 .
\end{aligned}
$$

Therefore, $j(x) \geq 0$ for all $0 \leq x \leq a$ and especially $j^{-}(0) \leq j^{+}(0)$. Furthermore, by (3.27), $p$ is nonincreasing and we just need to find an estimate on $p(0)=p^{+}(0)+p^{-}(0)$. 
The ingoing momentum flux $p^{+}(0)$ is given by the boundary condition at $x=0$, so it remains to estimate $p^{-}(0)$. By the Cauchy-Schwarz inequality,

$$
\begin{aligned}
p^{-}(0) & =\int_{\xi<0}|\xi|^{2} \mathrm{~d} \mu_{0}(v) \\
& \leq\left(\int_{\xi<0}|\xi| \mathrm{d} \mu_{0}(v)\right)^{1 / 2}\left(\int_{\xi<0}|\xi|^{3} \mathrm{~d} \mu_{0}(v)\right)^{1 / 2} \\
& \leq\left(j^{+}(0)\right)^{1 / 2}\left(q^{-}(0)\right)^{1 / 2}
\end{aligned}
$$

where $q^{+}(x)=\int_{\xi>0} \xi v^{2} \mathrm{~d} \mu_{x}(v), q^{-}(x)=\int_{\xi<0}|\xi| v^{2} \mathrm{~d} \mu_{x}(v)$ and $q(x)=q^{+}(x)-q^{-}(x)$. Furthermore, the energy flux $q$ fulfills

$$
\frac{\mathrm{d} q}{\mathrm{~d} x}=(\lambda-1) \tau \rho e \leq 0, \quad e=\int v^{2} \mathrm{~d} \mu_{x}(v)
$$

and

Hence, from (3.28)

$$
q^{+}(a)+q^{-}(0) \leq q^{+}(0)+q^{-}(a) .
$$

$$
p^{-}(0) \leq\left(j^{+}(0)\right)^{1 / 2}\left(q^{+}(0)+q^{-}(a)\right)^{1 / 2}
$$

Now

$$
q^{-}(a) \leq c_{2} j^{+}(a)
$$

because of (3.26) and

$$
j^{+}(a) \leq \frac{1}{c_{1}} p^{+}(a)
$$

because of the "spreading condition" (3.25). Using (3.29), (3.30) and $p(a) \leq p(0)$ yields

$$
p^{-}(0) \leq\left(j^{+}(0)\right)^{1 / 2}\left(q^{+}(0)+\frac{c_{2}}{c_{1}}\left(p^{+}(0)+p^{-}(0)\right)\right)^{1 / 2} \text {. }
$$

Recognizing that

$$
a b \leq \frac{1}{\varepsilon} a^{2}+\varepsilon b^{2}, \quad \forall \varepsilon, a, b>0,
$$

we get

$$
p^{-}(0) \leq \frac{1}{\varepsilon} j^{+}(0)+\varepsilon\left(q^{+}(0)+\frac{c_{2}}{c_{1}} p^{+}(0)\right)+\varepsilon \frac{c_{2}}{c_{1}} p^{-}(0), \quad \forall \varepsilon>0,
$$

from which we conclude that $p^{-}(0)$ is bounded.

We consider the problem (3.15) together with the (partial) diffusive boundary conditions

$$
\begin{aligned}
& \left.\mu_{0}\right|_{\{\xi>0\}}=\int_{\xi^{\prime}<0} m_{\beta_{0}}(v) \frac{\left|\xi^{\prime}\right|}{|\xi|} \mathrm{d} \mu_{0}(v) \\
& \left.\mu_{a}\right|_{\{\xi<0\}}=\int_{\xi^{\prime}>0} R_{a}\left(v^{\prime} \rightarrow v\right) \frac{\left|\xi^{\prime}\right|}{|\xi|} \mathrm{d} \mu_{a}(v)
\end{aligned}
$$

Here, $m_{0}(v)$ is a normalized Maxwellian with (inverse) temperature $\beta_{0}$ as given in (3.11) and $R_{a}$ should satisfy the conditions (3.23)-(3.26): This is indeed the case for the conditions (3.9) and (3.10) as pointed out in Ref. [6]. 
Theorem 3.5 For any $\delta>0$, the problem (3.15) together with the boundary conditions (3.31), (3.32) has a one parameter family of measure solutions $\mu . \in X$. These solutions can be parametrized by the outgoing mass flux at $x=0$.

\section{Proof}

Let $\mu^{?}$ be as in Lemma 3.4, then

$$
\begin{aligned}
\left\|\mu^{\tau}\right\| & \leq \sup _{x \in[0, a]}\left(\int_{\substack{0<\xi<\delta \\
1 / \delta<\xi}} \mathrm{d} \mu_{0}^{+}+\int_{\substack{-\delta<\xi<0 \\
-1 / \delta>\xi}} \int_{\xi^{\prime}>0} R_{a}\left(v^{\prime} \rightarrow v\right) \frac{\left|\xi^{\prime}\right|}{|\xi|} \mathrm{d} \mu_{0}^{+} \mathrm{d} v+\frac{1}{\delta^{2}} \int \xi^{2} \mathrm{~d} \mu_{x}^{\tau}\right) \\
& \leq C_{1}(\delta) .
\end{aligned}
$$

Here, $C_{1}(\delta)$ is a constant which depends on the boundary values and on $\delta$, but not on $\tau$ and $R$. Then, by the same argument as in Ref. [2], one concludes that for $\tau_{1}=4 \pi C(\delta)$ there exists a fixed point $\mu^{\tau_{1}}$ of $T\left(\tau_{1}\right)$, i.e. $T\left(\tau_{1}\right) \mu^{\tau_{1}}=\mu^{\tau_{1}}$.

Now we consider at $x=0$ the (prescribed) measure $\mu_{0}^{+}$with density $\tilde{j}^{-}(0) m_{0}(v)$, where $\tilde{j}^{-}(0)$ is some positive constant and $m_{0}$ is defined as in (3.31). What remains to show is that the corresponding fixed point $\mu^{\tau_{1}}$ fulfills the condition

$$
\int_{\xi<0}|\xi| \mathrm{d} \mu_{0}=\tilde{j}^{-}(0) .
$$

This can be done with the same arguments as in the previous subsection. Because $\mu^{T_{1}}$ is a fixed point of $T\left(\tau_{1}\right)$, we get by mass conservation the equation

$$
\frac{\mathrm{d} j}{\mathrm{~d} x}=0,
$$

where $j(x)=\int \xi \mathrm{d} \mu_{x}^{\tau_{1}}$. At $x=a$ we have, because of the mass conservation of the boundary kernel, $j(a)=0$, from which we conclude that $j(x)=0$ for all $x \in[0, a]$. Hence, at $x=0$, this yields

$$
j^{-}(0)=\int_{\xi<0}|\xi| \mathrm{d} \mu_{0}^{\dot{\tau}_{1}}=\int_{\xi>0} \xi \mathrm{d} \mu_{0}^{+} .
$$

Because $\mu_{0}^{+}$is the measure with density $\tilde{j}^{-}(0) m_{0}(v)$, we.get

$$
j^{-}(0)=\int_{\xi>0} j^{-}(0) \xi m_{0}(v) \mathrm{d} v=\tilde{j}^{-}(0) .
$$

Hence, the fixed point $\mu_{0}^{\tau_{1}}$ is a solution which fulfills the boundary conditions (3.31), (3.32).

Moreover, because $j^{-}(0)$ is some positive constant, there exists a one parameter family of solutions for fixed boundary conditions (defined by the (inverse) temperature $\beta_{0}$ and the boundary kernel $R_{a}$ ) and $j^{-}(0)$ parametrizes this family.

Remark 3.6 The result given in Theorem 3.5 is more general than the problem formulated at the beginning, i.e. the steady Boltzmann equation (3.8) together with the boundary conditions (3.9), (3.10). At $x=a$ an arbitrary boundary kernel $R_{a}$ which satisfies the conditions $(3.23)-(3.26)$ can be used. 


\section{A-Priori Estimates for the Steady Boltzmann Equation}

We consider the steady Boltzmann equation for $f=f(x, v)$

$$
v \nabla_{x} f=Q(f)
$$

on the bounded domain $\Omega \subset \mathbb{R}^{n}, n=1,2$ or 3 . Let $v=(\xi, \eta, \zeta)$, then the invariants of equation (4.1) are

$$
\operatorname{div}\left(\int v f(x, v) \mathrm{d} v\right)=0
$$

(mass conservation),

$$
\operatorname{div}\left(\int v w f(x, v) \mathrm{d} v\right)=0
$$

for $w \in\{\xi, \eta, \zeta\}$ (momentum conservation) and

$$
\operatorname{div}\left(\int v v^{2} f(x, v) \mathrm{d} v\right)=0
$$

(energy conservation).

Integrating (4.2) and applying the divergence theorem yields

$$
\int_{\partial \Omega} \int_{v \cdot n<0}|v \cdot n(x)| f(x, v) \mathrm{d} v \mathrm{~d} \sigma(x)=\iint_{\partial \Omega}|v \cdot n(x)| f(x, v) \mathrm{d} v \mathrm{~d} \sigma(x),
$$

where the term on the right hand side is given if the ingoing flux is precribed as boundary condition. The same holds taking the energy equation (4.4), yielding

$$
\int_{\theta \Omega} \int_{v \cdot n<0}|v \cdot n(x)| v^{2} f(x, v) \mathrm{d} v \mathrm{~d} \sigma(x)=\int_{\theta \Omega} \int_{v \cdot n>0}|v \cdot n(x)| v^{2} f(x, v) \mathrm{d} v \mathrm{~d} \sigma(x) .
$$

This proves

Proposition 4.1 Consider equation (4.1) together with a boundary condition $f(x, v)=\Phi(x, v)$ for $x \in \partial \Omega, v \cdot n(x)>0$ and assume that the ingoing mass and energy flux are bounded. Then, for every solution $f(x, v)$ of (4.1),

$$
\begin{gathered}
\int_{S} \int_{v \cdot n<0}|v \cdot n(x)| f(x, v) \mathrm{d} v \mathrm{~d} \sigma(x) \leq c_{1} \\
\int_{S} \int_{v \cdot n<0}|v \cdot n(x)| v^{2} f(x, v) \mathrm{d} v \mathrm{~d} \sigma(x) \leq c_{2}
\end{gathered}
$$

for all $S \subset \partial \Omega$ measurable. Here, $c_{1}$ and $c_{2}$ are two constants which depend only on the boundary values.

In Ref. [7] the conservation quantities (4.2)-(4.4) were used to prove a-priori estimates for general steady discrete-velocity models in two space dimensions. The solutions of the discrete model on a bounded domain $\Omega \subset \mathbb{R}^{2}$ - with prescribed fluxes at the boundary - were estimated in terms of the boundary values by integrating the solution along a line which bisects $\Omega$ into two subdomains. 
This result can be generalized to the full, 2- or 3-dimensional boundary value problem for the steady Boltzmann equation: let $H_{0}^{\prime}$ be an arbitrary hyperplane in $\mathbb{R}^{n}$ such that $H_{0}=H_{0}^{\prime} \cap \Omega \neq \emptyset$ and $n_{0}$ be the normal vector on $H_{0}$,

$$
n_{0}=a \cdot i+b \cdot j+c \cdot k .
$$

Multiplying (4.3) for $w=\xi$ by $a$, for $w=\eta$ by $b$ and for $w=\zeta$ by $c$ yields

$$
\operatorname{div}\left(\int\left(v \cdot n_{0}\right) v f(x, v) \mathrm{d} v\right)=0
$$

Let $x_{0} \in H^{\prime} \cap \Omega$ be an arbitrary but fixed reference point. Applying the divergence theorem on the domain $\Omega_{1}=\left\{x \in \Omega ;\left(x-x_{0}\right) \cdot n_{0}>0\right\}$ (i.e., the part of $\Omega$ on one side of $H^{\prime}$ ), we get

$$
\int_{H_{0}} \int_{\boldsymbol{R}^{3}}\left(v \cdot n_{0}\right)^{2} f(x, v) \mathrm{d} v \mathrm{~d} \sigma(x)+\int_{\partial \Omega_{1}} \int_{\boldsymbol{R}^{3}}\left(v \cdot n_{0}\right)(v \cdot n(x)) f(x, v) \mathrm{d} v \mathrm{~d} \sigma(x)=0
$$

with $\partial \Omega_{1}=\partial \Omega \cap \bar{\Omega}_{1}$. Hence,

$$
\begin{aligned}
\int_{H_{0}} \int_{R^{3}}\left(v \cdot n_{0}\right)^{2} f(x, v) \mathrm{d} v \mathrm{~d} \sigma(x)= & -\int_{\partial \Omega_{1}} \int_{v \cdot n(x)>0}\left(v \cdot n_{0}\right)(v \cdot n(x)) f(x, v) \mathrm{d} v \mathrm{~d} \sigma(x) \\
& -\int_{\partial \Omega_{1}} \int_{v \cdot n(x)<0}\left(v \cdot n_{0}\right)(v \cdot n(x)) f(x, v) \mathrm{d} v \mathrm{~d} \sigma(x) .
\end{aligned}
$$

The first term on the right hand side of (4.7) is controlled by the ingoing flux at $\partial \Omega$. The second term can be estimated as follows:

$$
\begin{aligned}
\int_{\partial \Omega_{1}} \int_{v \cdot n(x)<0}\left|v \cdot n_{0} \| v \cdot n(x)\right| f(x, v) \mathrm{d} v \mathrm{~d} \sigma(x) & \leq \int_{\partial \Omega_{1}} \int_{v \cdot n(x)<0}|v \cdot n(x)||v| f(x, v) \mathrm{d} v \mathrm{~d} \sigma(x) \\
& \leq \int_{\partial \Omega_{1}} \int_{v \cdot n(x)<0}|v \cdot n(x)| f(x, v) \mathrm{d} v \mathrm{~d} \sigma(x) \\
& +\int_{\partial \Omega_{1}} \int_{v \cdot n(x)<0}|v \cdot n(x)| v^{2} f(x, v) \mathrm{d} v \mathrm{~d} \sigma(x)
\end{aligned}
$$

and the last two terms are bounded in terms of the ingoing mass and energy flux by (4.5) and (4.6). Hence we have

Theorem 4.2 Consider the steady Boltzmann equation (4.1) with boundary condition $f(x, v)=$ $\Phi(x, v)$ such that the ingoing mass and energy flux are bounded. Let $H_{0}^{\prime}$ be a hyperplane with normal vector $n_{0}$ and $H_{0}=H_{0}^{\prime} \cap \Omega \neq \emptyset$. Then, for every solution $f(x, v)$ of. (4.1),

$$
\int_{H_{0}} \int_{\mathbb{R}^{3}}\left(v \cdot n_{0}\right)^{2} f(x, v) \mathrm{d} v \leq C,
$$

where $C$ is a constant which depend only on the boundary values. 


\section{Numerical Examples}

While the theoretical investigation of the Boltzmann equation still offers a interesting variety of open problems, e.g., the existence and uniqueness of solution for the multidimensional steady Boltzmann equation with arbitrary boundary conditions, during the last decade, numerical simulation methods became efficient tools for the prediction of rarefied gas flows for real applications, e.g., the description of the reentry path of space vehicles. We refer the reader to Ref. [8].

In the following, two examples for the numerical simulation of boundary value problems are given. An elaborated version of the numerical studies can be found in Ref. [9]. A detailed description of the particle method used is given in Ref. [10].

\subsection{One-Dimensional Slab Geometry}

We consider the steady Boltzmann equation in the slab $0 \leq x \leq 1$,

$$
\xi \frac{\mathrm{d} f}{\mathrm{~d} x}=Q(f)
$$

where the boundary conditions are a prescribed (Maxwellian) flux at $x=0$ and a diffusive boundary condition at $x=1$.

For the simulation we use an instationary particle method which solves the corresponding time-dependent version of (5.1).

Remark 5.1 Particle methods for the Boltzmann equation are in general simulations of instationary problems. Recently it was shown how to derive a particle scheme directly for the stationary equation [11].

It is shown in the following that the result of Subsection 3.2 is rediscovered in the numerical simulation. It is expected, that - in the stationary state - the outgoing mass flux at $x=0$ will balance the ingoing flux prescribed by the boundary condition at $x=0$.

For the numerical simulation we use the dimensionless form of (5.1) and the following set of numerical parameters: the mean free path is assumed to be 0.1 compared to the slab length equal to 1 which results in a Knudsen number of 0.1 . The (monatomic) gas interactions are modelled by the hard-sphere gas, i.e. $B\left(\left|v-v_{*}\right|, n\right)=\left(v-v_{*}, n\right)$.

The (scaled) temperature of the ingoing flux is 0.2 , the temperature for the diffusive boundary condition at $x=1$ equal to 2 . Initially, the gas is assumed to be in an equilibrium with temperature 1 and zero mean velocity.

The slab is divided into. 20 equisized cells and in every cell the density is assumed to be homogeneous and 4000 particles per cell are used to approximate the density function at time $t=0$. The boundary condition at $x=0$ is realized by adding an artificial cell at the left side of the slab which is filled - in every time step - with 8000 particles according to a Maxwellian distribution with temperature $\hat{0} . \hat{2}$ and zero mean velocity. 


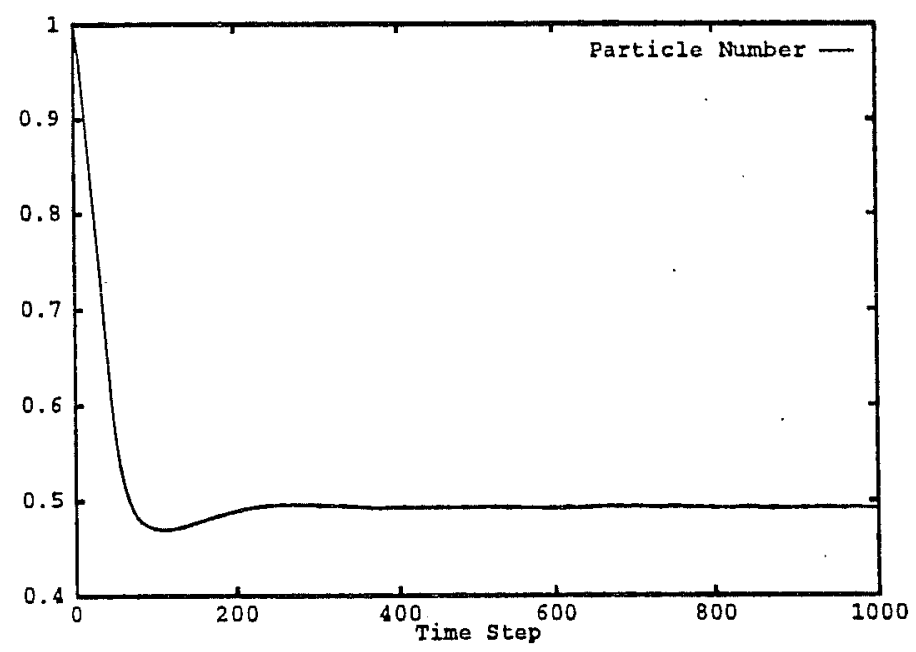

Fig. 5.1. Instationary Total Particle Number.

Figure 1 presents the instationary behaviour of the (normalized) total particle number in the slab. It can be seen that after nearly 500 time steps this quantity becomes stationary at about $1 / 2$ of the initial value. The results are obtained using 20 independent samples.

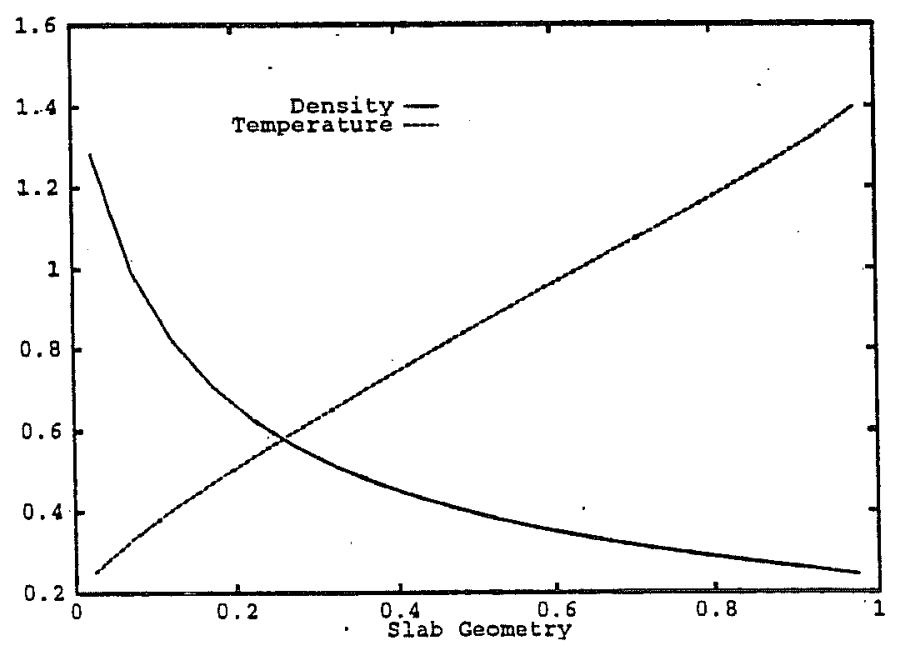

Fig. 5.2. Density and Temperature Profle along $0 \leq x \leq 1$.

Typical stationary solution profiles are shown in Fig. 2, namely the density and the temperature. 


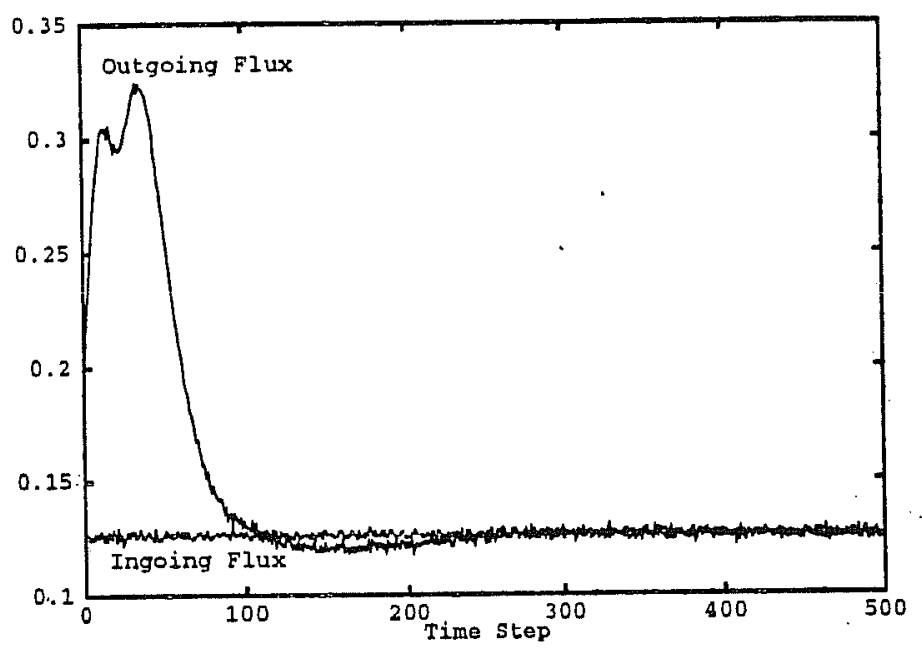

Fig. 5.3. Instationary In- and Outgoing Mass Flux at $x=0$.

Finally, Fig. 3 shows the (normalized) in- and outgoing mass flux at $x=0$. At the beginning of the simulation the outgoing flux increases up to a maximal value and drops down later on in the stationary state - to the ingoing mass flux prescribed by the time-independent boundary conditions. Due to the overshoot of the outgoing mass flux the total particle number drops down to the stationary value.

Changing the free parameter of the problem, the outgoing mass flux, will produce a different solution. We refer the reader to Ref. [9].

Remark 5.2 If the boundary condition at $x=0$ would be diffusive with the same temperature as above, due to mass conservation, the particle number would remain constant. In this case, the total mass described by the initial condition is the free parameter of the problem.

\subsection{A Two-Dimensional Problem}

In this section we present one example for a two-dimensional problem on the square $[0,1]^{2}$, i.e. we consider the two-dimensional Boltzmann equation

$$
\xi \frac{\partial f}{\partial x}+\eta \frac{\partial f}{\partial y}=Q(f)
$$

with the periodic boundary conditions

$$
\begin{aligned}
& f(x, 0, v)=f(x, \hat{1}, v), \quad \eta>0, \quad \hat{0} \leq x \leq 1 \\
& f(x, 1, v)=f(x, 0, v), \quad \eta<0, \quad 0 \leq x \leq 1
\end{aligned}
$$

and the diffusive boundary conditions

$$
\begin{array}{rlll}
-\xi f(0, y, v) & =j^{-}(y) m_{\alpha(y)}, & \xi<0, & 0<y<1 \\
\xi f(1, y, v) & =j^{+}(y) m_{\gamma(y)}, & \xi>0, & 0<y<1
\end{array}
$$


where

$$
\begin{aligned}
& \alpha(y)= \begin{cases}\beta_{1} & \text { if } 0 \leq y<\frac{1}{4} \text { or } \frac{1}{2} \leq y<\frac{3}{4} \\
\beta_{0} & \text { if } \frac{1}{4} \leq y<\frac{1}{2} \text { or } \frac{3}{4} \leq y \leq 1\end{cases} \\
& \gamma(y)=\left\{\begin{array}{ll}
\beta_{0} & \text { if } 0 \leq y<\frac{1}{4} \text { or } \frac{1}{2} \leq y<\frac{3}{4} \\
\beta_{1} & \text { if } \frac{1}{4} \leq y<\frac{1}{2} \text { or } \frac{3}{4} \leq y \leq 1
\end{array},\right.
\end{aligned}
$$

$\beta_{0}$ and $\beta_{1}$ are two given (inverse) temperatures and $j^{-}(y)\left(j^{+}(y)\right)$ the $y$-dependent outgoing mass flux at $x=0(x=1)$.

For the following example $\beta_{0}=0.2, \beta_{1}=2$, and the mean free path is equal to 0.1 . The domain $[0,1]^{2}$ is divided into 400 small squares and 200 particles per square are used to approximate the initial condition. The binary collisions are modelled by the hard-sphere interaction law.

Figure 5.4 shows the density profile in the stationary state obtained after 1000 time steps and 500 time steps to average the result. The corresponding temperature profile is given in Fig. 5.5.

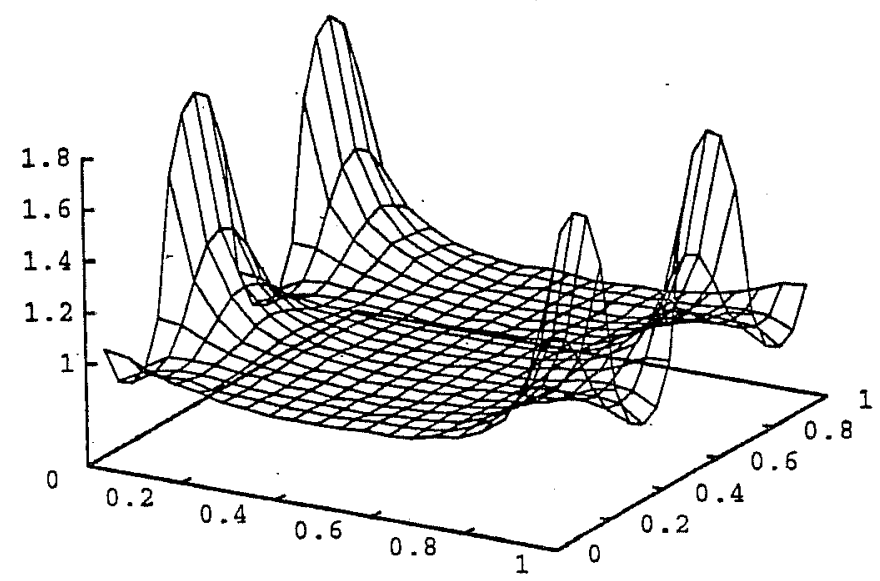

Fig. 5.4. Density Profile. 


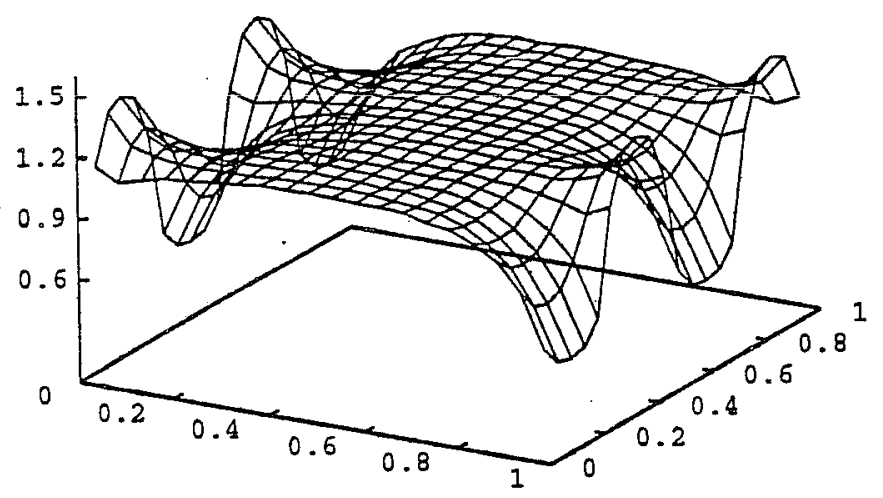

Fig. 5.5. Temperature Profile.

Acknowledgement: This research was supported by the Natural Sciences and Engineering Research Council of Canada under grant Nr. A 7847. Jens Struckmeier would like to acknowledge the hospitality of the Department of Mathematics and Statistics at the University of Victoria, where this research was done.

\section{References}

[1] Kaniel, S. and Shinbrot, M.: The Boltzmann Equation, I: Uniqueness and Global Existence, Comm. Math. Phys. 59, 65-84 (1978).

[2] Arkeryd, L., Cercignani, C. and Illner, R.: Measure Solutions of the Steady Boltzmann Equation in a Slab, Commun. Math. Phys. 142, 285-296 (1991).

[3] Cercignani, C., Illner, R. and Shinbrot, M.: A Boundary Value Problem for Discrete-Velocity Models, Duke Math. J. 55, 889-900 (1987).

[4] Arkeryd, L. and Nouri, A.: Asymptotics of the Boltzmann Equation with Diffuse Reflection Boundary Conditions, preprint.

[5] Cercignani, C. and Illner, R.: Global Weak Solutions of the Boltzmanin equation in a Slab with Diffusive Boundary Conditions, to appear in Arch. Rat. Mech. Anal..

[6] Arkeryd, L. and Maslova, N.: On Diffuse Reflection at the Boundary for the Boltzmann Equation and Related Equations, J. of Stat. Phys. 77, 1051-1077 (1994).

[7] Illner, R.: On Steady Boundary Value Problems in Discrete Kinetic Theory, and its Application to Digital Image Processing, in: Monaco, R. (Ed.), Discrete Kinetic Theory, Lattice Gas Dynamics and Foundations of Hydrodynamic, World Scientific, 178-191 (1989). 
[8] Shizgal, B.D. and Weaver, D.P. (Eds.), Rarefied Gas Dynamics, Progress in Astronautics and Aeronautics, Vols. 159 and 160, AIAA, Washington (1994).

[9] Struckmeier, J.: Simulation of Boundary Value Problems for the Boltzmann Equation, in preparation.

[10] Neunzert, H. and Struckmeier, J.: Particle Methods for the Boltzmann Equation, ACTA NUMERICA 1995, Cambridge, 417-457 (1995).

[11] Bobylev, A.V. and Struckmeier, J.: Numerical Simulation of the Stationary, One-Dimensional Boltzmann Equation by Particle Methods, preprint, to appear in Eur. J. of Mech. B/Fluids. 